\title{
Editorial
}

\section{Italian Research Facilities for Fundamental Physics}

\author{
Marco Selvi $^{1}$ (D) and Francesco Terranova ${ }^{2, *(D)}$ \\ 1 INFN sez. di Bologna, Viale C. Berti Pichat 6/2, I-40127 Bologna, Italy; marco.selvi@bo.infn.it \\ 2 Department of Physics, University of Milano-Bicocca and INFN sez. di Milano-Bicocca, Piazza della Scienza 3 , \\ I-20126 Milano, Italy \\ * Correspondence: francesco.terranova@cern.ch
}

check for updates

Citation: Selvi, M.; Terranova, F.

Italian Research Facilities for

Fundamental Physics. Universe 2022,

8, 82. https://doi.org/

10.3390/universe8020082

Received: 23 January 2022

Accepted: 25 January 2022

Published: 27 January 2022

Publisher's Note: MDPI stays neutral with regard to jurisdictional claims in published maps and institutional affiliations.

Copyright: (c) 2022 by the authors Licensee MDPI, Basel, Switzerland. This article is an open access article distributed under the terms and conditions of the Creative Commons Attribution (CC BY) license (https:// creativecommons.org/licenses/by/ $4.0 /)$.
This Special Issue of Universe addresses the international community working at the Italian Research Facilities for Fundamental Physics, Italian labs and facilities playing a pivotal role in the core fields of this journal, such as gravitational waves, dark matter and rare event searches, neutrino astronomy, and underground physics. The experiments hosted at LNGS (Laboratori Nazionali del Gran Sasso), LNS/KM3NeT (Laboratori Nazionali del Sud), LNF (Laboratori Nazionali di Frascati), LNL (Laboratori Nazionali di Legnaro), and the EGO (European Gravitational Observatory) are among the most sensitive detectors in the world, fostering a broad community of experimentalists and theoreticians. The twofold goal of this Special Issue is to review the most important discoveries achieved in these research centers in the last few years and discuss future perspectives, a particularly timely task since the results of observational cosmology and high-energy accelerators have completely reshaped our research aims and raised new questions requiring the development of novel approaches and experimental techniques, representing both a challenge and an opportunity for existing facilities to broaden their research scope and blur the traditional boundaries among disciplines.

Here, we introduce the papers belonging to the Special Issue, and would like to take the opportunity to thank all authors and colleagues who helped us in the preparation of this collection. Their ideas, suggestions, and contributions made this Special Issue an effective tool for highlighting the results and perspectives of the Italian physics community.

\section{- Astroparticle Physics at INFN}

The Italian Institute for Nuclear Research (INFN), Italy, has been a key contributor in the development of particle, astroparticle, nuclear, and fundamental physics, running major laboratories, employing researchers and engineers leading those fields, and fully integrating with Italian universities and their research programs. At the global level, the INFN is one of the most important funding agencies supporting research at all major labs in the world, including CERN (EU), Fermilab (US), KEK (Japan), and IHEP (China), with astroparticle physics coordinated by a special committee (Commissione Scientifica Nazionale II) led by O. Cremonesi. The paper by Cremonesi [1] introduces our Special Issue with a document reviewing INFN's contributions to astroparticle science, including supported experimental activities such as the study of cosmic radiation, the dark universe, general and quantum physics, neutrino properties, and the search for gravitational waves. Dr. Cremonesi [1] is also a renowned expert in the field of neutrino physics and, in particular, neutrinoless double beta decay, and the experiment he led up to 2018 (CUORE), its upgrade (CUPID), and future projects are nicely reviewed as well.

\section{- Advanced Virgo: Status of the Detector, Latest Results, and Future Prospects}

The discovery of gravitational waves has been a breakthrough in the fields of astroparticles and cosmology and the culmination of many decades of research. From its first scientific run in 2007, the Virgo detector in Italy has constantly been upgraded over the years, and, since 2017, with the Advanced Virgo project, the detector reached a high sensitivity, allowing the detection of several classes of sources and investigations of new physics. 
The combination of Ligo and Virgo opened up the era of multi-messenger physics due to the precision of the combined interferometers pinning down the location of the gravitational wave source with exquisite precision, providing a gravitational signature to celestial objects identified by telescopes and cosmic ray experiments. The paper by D. Bersanetti et al. [2] highlights such a success story, reporting the main hardware upgrades of the detector and the main astrophysical results in the last five years. The authors are distinguished members of the Virgo collaboration involved, in particular, in the design and construction of the Advanced Virgo project, and provide the readers of Universe the rationale of the Virgo upgrades, a detailed description of the Virgo results, and a well-thought-out assessment of its potential in the post-discovery era.

\section{- GERDA and LEGEND: Probing the Neutrino Nature and Mass at $100 \mathrm{meV}$ and Beyond}

Neutrinoless double beta decay plays a vital role in Italy, since several leading experiments have been hosted at the Gran Sasso laboratories of the INFN. The GERDA (GERmanium Detector Array) project, located at LNGS, was started in 2005, a few years after the claim of evidence for the neutrinoless double beta decay $(0 v \beta \beta)$ of ${ }^{76} \mathrm{Ge}$ to the ground state of ${ }^{76}$ Se. Both the GERDA setup and the analysis tools evolved, along with the project's lifetime, allowed the achievement of the remarkable average energy resolution of 3.6 and $2.6 \mathrm{keV}$ for Coaxial Germanium (COAX) detectors and for Broad Energy Germanium (BEGE), respectively. GERDA achieved an outstanding background index, paving the way to the next-generation experiment-LEGEND. GERDA's performances in terms of the background index, energy resolution, and exposure are the best achieved so far by ${ }^{76}$ Ge double beta decay experiments, and in phase II, GERDA succeeded in operating in a background-free regime, setting a world record. In 2017, the LEGEND Collaboration was born from the merging of the GERDA and MAJORANA Collaborations and resources, with the aim of further improving GERDA's sensitivity. C. Cattadori and F. Salamida [3] describe the GERDA experiment, its evolution, the data analysis flow, a selection of its results and technological achievements, and, finally, the design, features, and challenges of LEGEND.

\section{- The Xenon Road to Direct Detection of Dark Matter at LNGS: The XENON Project}

The paper by P. Di Gangi [4] presents reviews devoted to Xenondark matter experiments in Italy. Dark matter, with the direct search of it being one of the most active fields of experimental physics in the last few decades, is key in understanding the universe and a portal to the discovery of new physics beyond. The Standard Model of particle physics. Liquid xenon (LXe) detectors demonstrated the highest sensitivities to the main dark matter candidates (WIMPs), and the experiments of the XENON project, located at the LNGS (Italy), lead the field thanks to the dual-phase LXe time projection chamber technology. Since the first prototype, XENON10, built in 2005, each detector of the XENON project achieved the highest sensitivity to WIMP dark matter. XENON increased the LXe target mass by nearly a factor of 400 , up to the $5.9 \mathrm{t}$ of the current XENONnT detector installed at the LNGS in 2020. In this paper, the author reviews the principles of direct dark matter detection with LXe, detectors of the XENON project, challenges posed by background mitigation to ultra-low levels, and main results achieved by the XENON project in the search for dark matter.

\section{- Dark Matter Searches at LNF}

Dark matter detection relies on natural and artificial sources, its candidates able to be produced in well-controlled conditions using accelerators, unique tools for addressing the origin of dark matter. A broad class of candidates can be explored by small experiments with a short timescale and an investment affordable for national laboratories, such as the Frascati Laboratories of the INFN (LNF). This is the reason why a laboratory that, traditionally, focused on particle physics studies using accelerators, now hosts an important 
research program in this field. The paper by C. Gatti et al. [5] describes such a program, the expected sensitivity of the LNF experiments, and the technological challenges.

\section{- Optical Polarimetry for Fundamental Physics}

Sensitive magneto-optical polarimetry was proposed by E. Iacopini and E. Zavattini in 1979 for the detection of vacuum electrodynamic non-linearity, in particular, Vacuum Magnetic Birefringence (VMB), predicted in QED via the fluctuation of electron-positron virtual pairs, but can also be due to hypothetical axion-like particles (ALPs) and/or millicharged particles (MCP). Today ALPs are considered a strong candidate for dark matter and, as a consequence, the ALP search program complements the search for dark matter candidates in Italian research infrastructures. This program is reviewed by G. Zavattini and F. Della Valle in [6], broadening the scope of this Special Issue and covering the contributions of the INFN Legnaro Laboratories (LNL), also reviewing the latest proposals based on the achievements reached by Ferrara and CERN.

\section{- Borexino Results on Neutrinos from the Sun and Earth}

The final paper of this Special Issue is devoted to Borexino, an outstanding solar neutrino experiment hosted in Italy, completing data retrieval in 2021. Borexino is a 280ton liquid scintillator detector located at the LNGS in Italy, and since the start of its data retrieval in May 2007, it has provided several measurements of low-energy neutrinos from various sources. Borexino has also detected geoneutrinos, antineutrinos from the decays of long-lived radioactive elements inside the Earth, capable of being exploited as a new and unique tool to study our planet. The paper by Kumaran et al. [7] reviews the most recent Borexino results on solar energy and geoneutrinos, from highlighting the key elements of the analyses up to the discussion and interpretation of the results for neutrinos, solar energy, and geophysics.

Funding: This research received no external funding.

Conflicts of Interest: The authors declare no conflict of interest.

\section{References}

1. Cremonesi, O. Astroparticle physics at INFN. Universe 2021, 7, 224. [CrossRef]

2. Bersanetti, D.; Patricelli, B.; Piccinni, O.J.; Piergiovanni, F.; Salemi, F.; Sequino, V. Advanced Virgo: Status of the Detector, Latest Results and Future Prospects. Universe 2021, 7, 322. [CrossRef]

3. Cattadori, C.M.; Salamida, F. GERDA and LEGEND: Probing the Neutrino Nature and Mass at $100 \mathrm{meV}$ and beyond. Universe 2021, 7, 314. [CrossRef]

4. Di Gangi, P. The Xenon Road to Direct Detection of Dark Matter at LNGS: The XENON Project. Universe 2021, 7, 313. [CrossRef]

5. Gatti, C.; Gianotti, P.; Ligi, C.; Raggi, M.; Valente, P. Dark Matter Searches at LNF. Universe 2021, 7, 236. [CrossRef]

6. Zavattini, G.; Della Valle, F. Optical Polarimetry for Fundamental Physics. Universe 2021, 7, 252. [CrossRef]

7. Kumaran, S.; Ludhova, L.; Penek, O.; Settanta, G. Borexino Results on Neutrinos from the Sun and Earth. Universe 2021, 7, 231. [CrossRef] 